\title{
Robust Cassie State of Wetting in Transparent Superhydrophobic Coatings
}

\author{
Urandelger Tuvshindorj, ${ }^{\dagger, \dagger}$ Adem Yildirim, ${ }^{\dagger, \ddagger}$ Fahri Emre Ozturk, ${ }^{\dagger, \ddagger}$ and Mehmet Bayindir ${ }^{*}, \dagger, \ddagger, \S$ \\ ${ }^{\dagger}$ UNAM-National Nanotechnology Research Center, ${ }^{\ddagger}$ Institute of Materials Science and Nanotechnology, and ${ }^{\S}$ Department of \\ Physics, Bilkent University, 06800 Ankara, Turkey
}

\section{Supporting Information}

ABSTRACT: This paper investigates the stability of the Cassie state of wetting in transparent superhydrophobic coatings by comparing a single-layer microporous coating with a doublelayer micro/nanoporous coating. Increasing pressure resistance of superhydrophobic coatings is of interest for practical use because high external pressures may be exerted on surfaces during operation. The Cassie state stability against the external pressure of coatings was investigated by squeezing droplets sitting on surfaces with a hydrophobic plate. Droplets on the single-layer coating transformed to the Wenzel state and pinned to the surface after squeezing, whereas droplets on the
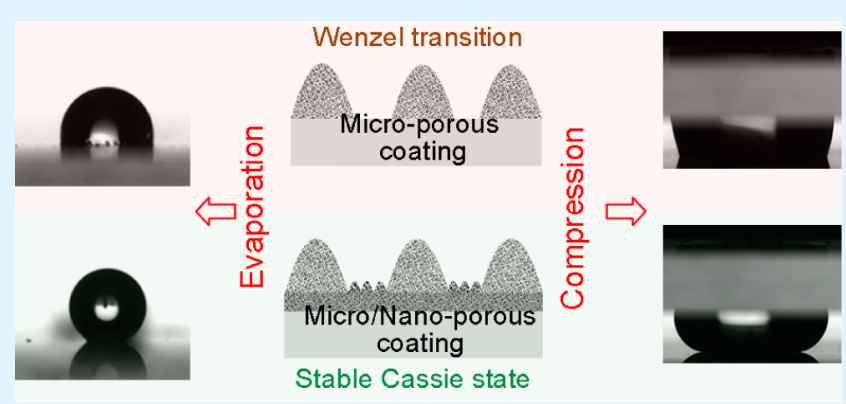
double-layer micro/nanoporous coating preserved the Cassie state and rolled off the surface easily. In addition, the contact angle and contact-line diameter of water droplets during evaporation from surfaces were in situ investigated to further understand the stability of coatings against Wenzel transition. A droplet on a microporous coating gradually transformed to the Wenzel state and lost its spherical shape as the droplet volume decreased (i.e., the internal pressure of the droplet increased). The contact line of the droplet during evaporation remained almost unchanged. In contrast, a water droplet on a double-layer surface preserved its spherical shape even at the last stages of the evaporation process, where pressure differences as high as a few thousand pascals were generated. For this case, the droplet contact line retracted during evaporation and the droplet recovered the initial water contact angle. The demonstrated method for the preparation of robust transparent superhydrophobic coatings is promising for outdoor applications such as self-cleaning cover glasses for solar cells and nonwetting windows.

KEYWORDS: Cassie state stability, superhydrophobic, self-cleaning, wettability, evaporation, organically modified silica

\section{INTRODUCTION}

A water droplet sitting on a superhydrophobic surface (i.e., the water contact angle is $>150^{\circ}$ ) can be in either the Wenzel or Cassie state depending on the morphology and chemistry of the surface. In the Wenzel state, the surface is completely wetted and the droplet pins to the surface, whereas in the Cassie state, the droplet wets the surface partially and air pockets form between the surface and water droplet. ${ }^{1-3}$ The Cassie state of wetting provides a large apparent water contact angle with low contact-angle hysteresis and results in a roll-off superhydrophobic surface, which is desired for practical applications such as self-cleaning windows and solar panels, ${ }^{4-6}$ underwater drag reduction, ${ }^{7,8}$ and anticorrosion coatings. ${ }^{9}$ Various successful methods have been developed by mimicking well-known examples from nature (e.g., Lotus leaves and butterfly wings) to prepare artificial superhydrophobic surfaces with the Cassie state of wetting using lithographic methods, ${ }^{10-12}$ sol-gel techniques, ${ }^{4,9,13-15}$ phase separation in polymer blends, ${ }^{16}$ electrospinning, ${ }^{17,18}$ and others. ${ }^{5,19-23}$ However, the Cassie state of wetting (roll-off) can easily and irreversibly transform to the Wenzel state of wetting (sticky) under external stimuli such as pressure, vibration, droplet impact, and droplet evaporation by complete filling of the air pockets with water. ${ }^{24-26}$ The poor stability of the Cassie state remains as an unsolved drawback against practical applications of superhydrophobic surfaces. ${ }^{26}$ Particularly, the applications that require outdoor or underwater operation, where surfaces are exposed to external conditions such as droplet impact or hydrostatic pressure, require a robust Cassie state. Several authors have investigated the parameters that affect the stability of the Cassie state in superhydrophobic coatings. ${ }^{5,26-40}$ However, in almost all of these studies, lithographically fabricated silicon or poly(dimethylsiloxane) (PDMS) pillars were used, ${ }^{26-30,32-36}$ which are not suitable for practical applications because of their high cost, limited material choice, and inefficiency for large-area fabrication. In addition, in none of these studies were fabricated surfaces optically transparent, which is a prerequisite for many applications of nonwetting coatings such as self-cleaning windows and solar cells in terms of the appearance and device performance. ${ }^{4,5}$ Therefore, facile fabrication methods for transparent superhydrophobic surfaces with a stable Cassie state of wetting are still needed.

\footnotetext{
Received: April 7, 2014

Accepted: May 13, 2014

Published: May 13, 2014
} 

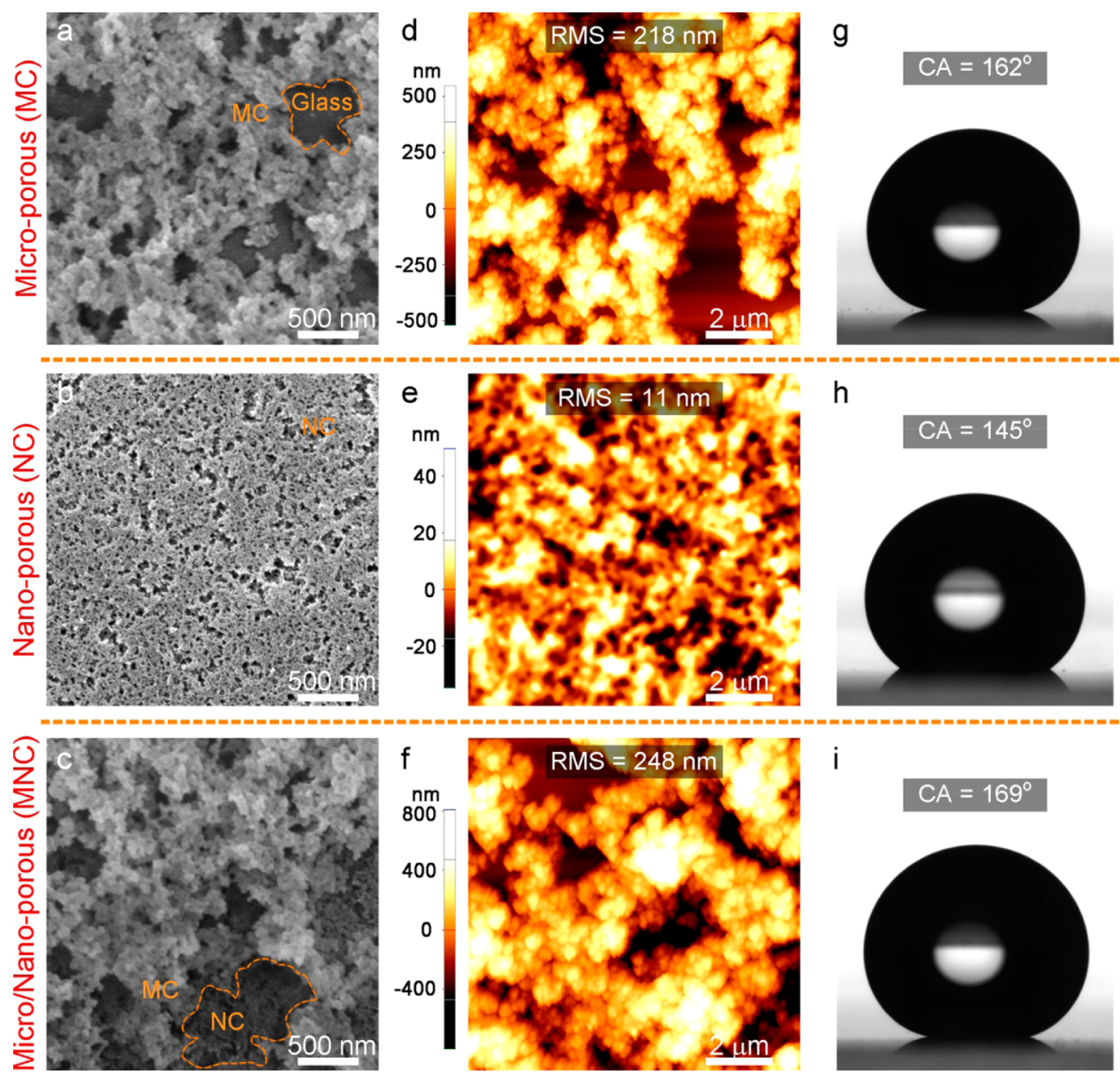

Figure 1. Characterization of the ormosil coatings. (a-c) SEM images of MC, NC, and MNC, respectively. The glass surface exposed through the openings in $\mathrm{MC}$ is outlined in orange. In the MNC, the bottom NC is outlined in orange. (d-f) AFM images of MC, NC, and MNC, respectively. (g-i) Photographs of water droplets sitting on MC, NC, and MNC, respectively.

Here, we investigated the effect of different levels of surface topography on the stability of the Cassie state of wetting in large-area and transparent superhydrophobic coatings. Three different organically modified silica (ormosil) coatings, (i) nanoporous hydrophobic coating (NC), (ii) microporous superhydrophobic coating (MC), and (iii) double-layer superhydrophobic coating with nanoporous bottom and microporous top layers (MNC), were prepared on glass surfaces. The stability of the Cassie state of coatings against the external pressure was examined by applying compression/relaxation cycles to water droplets sitting on the surfaces. The changes of the apparent contact angle, contact-angle hysteresis, and sliding-angle values of the surfaces before and after compression cycles were studied to determine the Cassie/ Wenzel transition behavior of the surfaces. In addition, water droplets were allowed to evaporate from the surfaces under ambient conditions, and the changes in the contact angles and contact-line diameters with increasing Laplace pressure were analyzed. We demonstrated that, upon combination of coatings with different levels of topography (i.e., MNC), it is possible to fabricate transparent superhydrophobic surfaces with extremely stable Cassie states of wetting on glass surfaces.

\section{EXPERIMENTAL SECTION}

Materials. Methyltrimethoxysilane (MTMS), oxalic acid, and ammonium hydroxide (25\%) were purchased from Merck (Germany), and dimethyl sulfoxide (DMSO) and methanol were purchased from Carlo-Erba (Italy). All chemicals were used as received.

Preparation of NC. Ormosil coatings were prepared according to our previous report. ${ }^{41}$ Initially, $1 \mathrm{~mL}$ of MTMS was dissolved in $2 \mathrm{~mL}$ of DMSO, and $0.5 \mathrm{~mL}$ of an oxalic acid solution $(10 \mathrm{mM})$ was slowly added to the mixture and stirred for $30 \mathrm{~min}$. Then $0.42 \mathrm{~mL}$ of an ammonia solution $(25 \%)$ and $0.19 \mathrm{~mL}$ of water in $5 \mathrm{~mL}$ of DMSO were added, and the solution was further stirred for $15 \mathrm{~min}$. Finally, the solution was left for gelation at $25{ }^{\circ} \mathrm{C}$. The gels are typically formed in about $1 \mathrm{~h}$. After gelation, approximately $20 \mathrm{~mL}$ of methanol was added onto the gels and incubated for at least $6 \mathrm{~h}$ at $25{ }^{\circ} \mathrm{C}$ to remove DMSO and unreacted chemicals. This treatment was repeated for four times to ensure complete removal of DMSO and other chemical residues. After washing, $12 \mathrm{~mL}$ of methanol was added onto the gels and gels were sonicated using an ultrasonic homogenizer for $45 \mathrm{~s}$ at $20 \mathrm{~W}$ to obtain ormosil colloids that are suitable for thin-film deposition. Then ormosil colloids were spin-coated on clean glass substrates at $2000 \mathrm{rpm}$ and dried at room temperature overnight. To increase the integrity and hydrophobicity, coatings were heat-treated at $450{ }^{\circ} \mathrm{C}$ for $1 \mathrm{~h}$.

Preparation of MC. The superhydrophobic microporous coatings were prepared according to our previous report. ${ }^{13}$ First, $1 \mathrm{~mL}$ of MTMS was dissolved in $9.74 \mathrm{~mL}$ of methanol. Then $0.5 \mathrm{~mL}$ of an oxalic acid solution $(1 \mathrm{mM})$ was added to the mixture and gently stirred for $30 \mathrm{~min}$. The mixture was left for hydrolysis for $24 \mathrm{~h}$ at room temperature. After hydrolysis, $0.42 \mathrm{~mL}$ of an ammonia solution $(25 \%)$ and $0.19 \mathrm{~mL}$ of water were added slowly to the mixture, and the reaction mixture was stirred for $15 \mathrm{~min}$. The solution was left for 2 days at $25{ }^{\circ} \mathrm{C}$ for gelation and aging. After aging of the gel network, 12 $\mathrm{mL}$ of methanol was added onto the gels and gels were sonicated using 
an ultrasonic homogenizer for $45 \mathrm{~s}$ at $20 \mathrm{~W}$ to obtain ormosil colloids. Then ormosil colloids were spin-coated on clean glass substrates at $2000 \mathrm{rpm}$ and dried at room temperature overnight. To increase the integrity and hydrophobicity, coatings were heat-treated at $450{ }^{\circ} \mathrm{C}$ for $1 \mathrm{~h}$.

Preparation of MNC. A double-layer coating was prepared by successive spin coating of ormosil colloids. Initially, a nanoporous layer (bottom layer) was spin-coated on clean glass substrates at $2000 \mathrm{rpm}$ and dried at room temperature overnight. Then a microporous layer (top layer) was spin-coated on the nanoporous layer at $2000 \mathrm{rpm}$ and dried at room temperature overnight. Finally, double-layer coatings were cured at $450{ }^{\circ} \mathrm{C}$ for $1 \mathrm{~h}$.

Characterization. The surface topography of the coatings was investigated with scanning electron microscopy (E-SEM; Quanta 200F, FEI) at high vacuum after coating with $5 \mathrm{~nm}$ of gold or platinum. Atomic force microscopy (AFM; XE-100E, psia) was used in noncontact mode to characterize the surface morphology and roughness of the coatings. Root-mean-square (rms) roughness values were calculated from three separate AFM images of the coatings, all of which were obtained from $10 \times 10 \mu \mathrm{m}^{2}$ area. The thicknesses of the coatings were measured with an ellipsometer (V-Vase, J. A. Woollam). Optical transmission experiments were performed with a UV-vis spectrophotometer (Cary 5000, Varian). Contact-angle measurements were performed using a contact-angle meter (OCA 30, Dataphysics). For static contact-angle measurements, $10 \mu \mathrm{L}$ of water droplets was used with the Laplace-Young fitting. Advancing and receding angles were measured by the addition and subtraction of $5 \mu \mathrm{L}$ of water to/ from the $10 \mu \mathrm{L}$ of droplets sitting on the surfaces. Sliding-angle measurements were performed using $10 \mu \mathrm{L}$ of water droplets.

\section{RESULTS AND DISCUSSION}

Preparation and Characterization of Ormosil Coatings. $\mathrm{MC}$ and $\mathrm{NC}$ transparent ormosil coatings on glass surfaces were prepared according to our previous reports. 13,41 MNCs were prepared by the subsequent coating of these two ormosil films: a dual-layer coating with $\mathrm{NC}$ as the bottom layer and $\mathrm{MC}$ as the top layer.

The first column of Figure 1 shows the SEM images of ormosil surfaces. MC demonstrated microporous and rough surface topography (Figure 1a). An underlying glass substrate can be observed through the openings of the coating (orange outlined area in Figure 1a). On the other hand, NC fully covers the surface and demonstrates nanoporous and uniform surface topography (Figure 1b). Lower-magnification SEM images are provided in the Supporting Information (SI; Figure S1) to demonstrate the uniformity of the coatings. Both layers of MNC coating can be clearly distinguished in the SEM image: rough microporous structure of $\mathrm{MC}$ at the top and nanoporous $\mathrm{NC}$ at the bottom (Figure 1c). Average surface roughness values of the coatings were calculated from their three separated AFM images $\left(10 \times 10 \mu \mathrm{m}^{2}\right)$ and are given in the second column of Figure 1 with the representative AFM images of the surfaces. As can be expected, MC and NC have high $(218 \pm 18$ $\mathrm{nm})$ and low $(11 \pm 2 \mathrm{~nm}) \mathrm{rms}$ surface roughness values, respectively. The surface roughness of double-layer $\mathrm{MNC}$ is on the same order with MC, $248 \pm 3 \mathrm{~nm}$.

Because of the low-energy surface methyl groups, coatings are intrinsically hydrophobic. In addition to the hydrophobic surface chemistry, their micro/nanostructured surfaces provide superhydrophobicity. The MC demonstrates roll-off superhydrophobicity (Cassie state) because of its rough and hydrophobic surface with a water contact angle of $162^{\circ}$ and a sliding angle of smaller than $1^{\circ}$ (Figure $1 \mathrm{~g}$ ). In comparison, even if the NC surface is also highly hydrophobic with a water contact angle of $145^{\circ}$, water droplets pinned to the surface (Wenzel state) because of its low surface roughness (Figure 1h). MNC demonstrated a Cassie state superhydrophobic property with water contact and sliding angles similar to those of the MC (Figure 1i).

Transparency of superhydrophobic coatings is desired for many practical applications including self-cleaning windows and solar panels. ${ }^{4-6,42-45}$ Figure 2 shows the transmission spectra

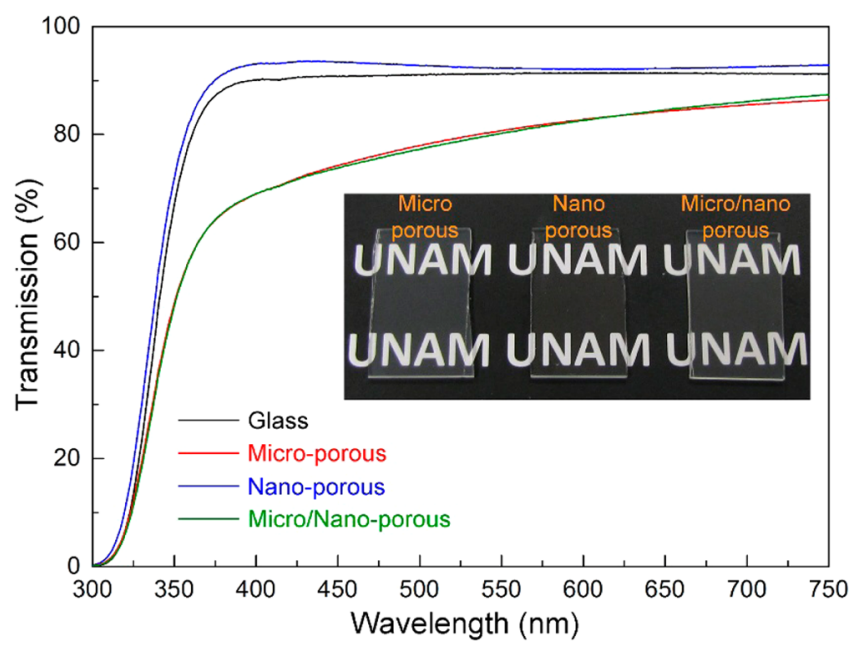

Figure 2. Transmission spectra of ormosil coatings and an uncoated glass substrate. All of the coatings are highly transparent at the visible wavelengths. The inset shows a photograph of transparent coatings.

of the ormosil coatings. Despite its high surface roughness value, MC demonstrates high transparency (up to $85 \%$ ) in the visible wavelengths. The slight decrease in the transmission compared to the bare glass is due to the light scattering from its microporous surface, which is more pronounced in smaller wavelengths. ${ }^{46}$ The transmission of NC is even higher than bare glass (i.e., antireflection property) because of its smooth surface and porous nature, which introduces a gradual refractive index change and reduces the reflectance of the glass. $4,41,47$ Lastly, the transmission spectrum of double-layer MNC is almost identical with that of MC because of the same scale of their surface porosity and roughness.

Compression Experiments. Squeezing water droplets between two surfaces is often used to investigate the stability of the Cassie state of wetting of superhydrophobic coatings against the external pressure. ${ }^{2,31,32,38}$ During compression of a droplet placed on a superhydrophobic surface, pressure differences as high as $300 \mathrm{~Pa}$ can be generated depending on the distance between two surfaces and the apparent contact angles of the surfaces. ${ }^{2}$ The pressure difference generated during compression can be calculated using the following Laplace equation: ${ }^{2}$

$$
\Delta P=\gamma\left(\cos \theta_{\mathrm{b}}+\cos \theta_{\mathrm{t}}\right) / x, \text { for } x<<R
$$

where $\gamma$ is the surface tension of water, $R$ is the radius of the droplet, $x$ is the gap between the top and bottom surfaces, and $\theta_{\mathrm{b}}$ and $\theta_{\mathrm{t}}$ are the water contact angles of the bottom and top surfaces, respectively.

For compression experiments, first we placed a droplet (10 $\mu \mathrm{L}$ ) on a NC surface (top plane) and slowly approached a bottom plate (MC or MNC) to squeeze the water droplet between two surfaces up to an interplanar distance $(\Delta x)$ of $\sim 0.4 \mathrm{~mm}$. Then we slowly relaxed the top surface. Figure 3a shows the frames of the water droplets during squeezing and relaxation between NC and MC (upper row) and NC and 

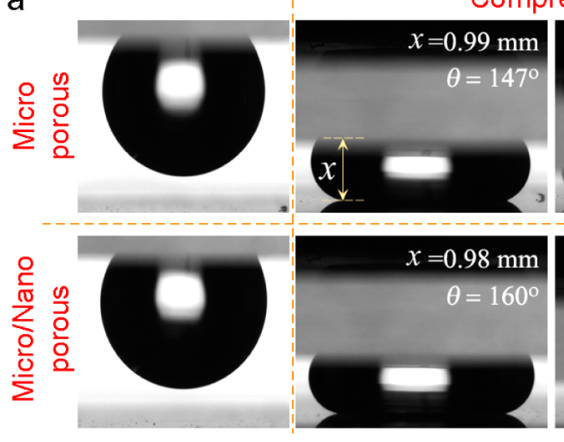

b

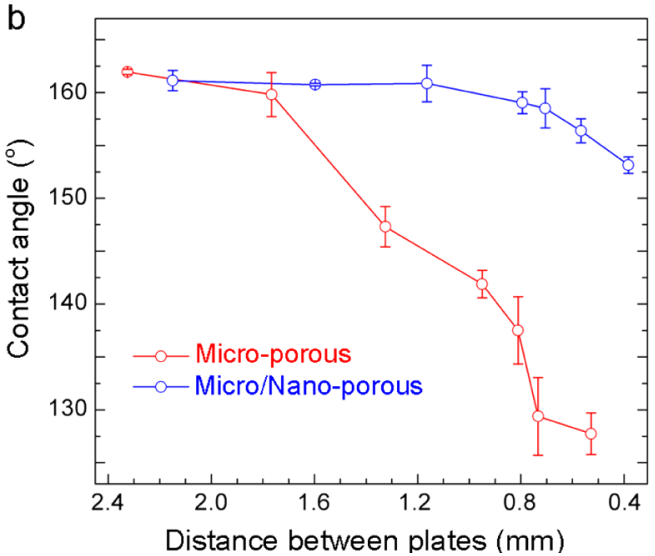

Compression
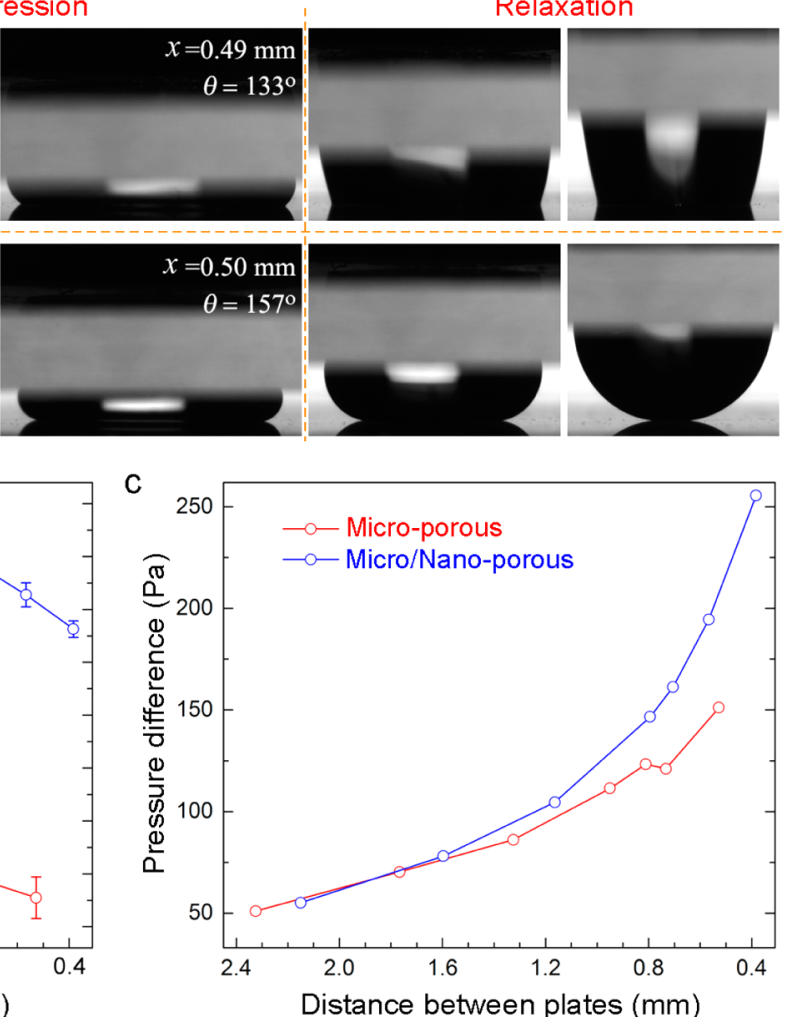

Figure 3. Compression experiments using the $\mathrm{NC}$ as the sticky hydrophobic top plate. (a) Photographs of the water droplets compressed and released between NC and MC (upper row) and NC and MNC (lower row). (b) Contact-angle change of coatings during compression. (c) Generated Laplace pressures for coatings during compression. Wenzel transition was observed for the MC at the first stages of compression; on the other hand, the Cassie state was preserved in the MNC even at the highest compression.

MNC (lower row). In the first stages of compression (the second column in Figure 3a), the bottom contact lines largely preserved their high water contact angle values for both MC and MNC. However, for the highest compression (the third column in Figure 3a), a significant decrease in the apparent contact angle of MC was observed, which suggests a transition between the Cassie and Wenzel states. ${ }^{2,31}$ On the other hand, double-layer MNC preserved the high water contact-angle value even at this high compression. The wetting difference between two coatings is more obvious during relaxation. It can be clearly seen from the fourth and fifth columns in Figure 3a that after compression a water droplet strongly sticks to the MC surface, indicating the Wenzel transition; quite the reverse, a droplet on the MNC surface relaxes easily without changing its spherical shape, indicating that the MNC surface preserved the Cassie state. ${ }^{31,32}$ Figure $3 \mathrm{~b}$ shows the in situ contact-angle measurements during compression of the surfaces, and Figure $3 c$ shows the generated pressure as a function of $\Delta x$. The contact angle of the MC sharply decreased below $150^{\circ}$ in the first stages of squeezing. On the other hand, the water contact angle of the MNC is larger than $150^{\circ}$ even at the lowest $\Delta x$ value. The generated Laplace pressure difference (eq 1) at highest compression (Figure 3c) is almost 2 -fold higher for the MNC because it demonstrates higher water contact-angle values than the MC during compression. From Figure 3b,c, it can be observed that, for the MC, Cassie to Wenzel transition occurred at a pressure difference of roughly around $80 \mathrm{~Pa}$, whereas no transition was observed for the MNC even at a pressure difference of $260 \mathrm{~Pa}$.
Besides the decrease in the apparent contact angle, increases in the contact-angle hysteresis and sliding angle are indicators of Cassie to Wenzel transition. ${ }^{48}$ Surfaces with the Cassie state of wetting demonstrate low contact-angle hysteresis values and smaller sliding-angle values due to easy roll-off of the droplets; however, for the Wenzel state of wetting, droplets pin to the surfaces with high contact-angle hysteresis values. To measure the water contact and sliding angles and contact-angle hysteresis of MC and MNC after droplet relaxation, we designed a compression experiment where a water droplet (10 $\mu \mathrm{L}$ ) is squeezed between two identical surfaces (MC or MNC; Figure 4, inset) and released. Figure 4 shows the contact angles of surfaces after release from a compression distance of $1 \mathrm{~mm}$. The contact angle of a released droplet on the MC sharply decreases to $127^{\circ}$, which is in good accordance with initial compression experiments. In contrast, there is only $5^{\circ}$ difference in the contact angles of the MNC before and after squeezing. Figure 5a shows the water droplet sliding angles before and after the compression test. Before squeezing, both surfaces were highly water repellent with sliding angles lower than $1^{\circ}$. However, a droplet on the MC strongly sticks to the surface after pressure release, and it remains pinned even at $90^{\circ}$ tilting. For the MNC, although an increase in the sliding angle after pressure release was observed, the droplet can easily roll off from the surface at $15^{\circ}$ of tilting. Figure $5 \mathrm{~b}$ shows the contact-angle hysteresis of the surfaces before and after compression. Both surfaces have contact-angle hysteresis values of around $2^{\circ}$ before compression. After compression, the increase in the contact-angle hysteresis is much more pronounced for $\mathrm{MC}\left(\sim 11^{\circ}\right)$ than for $\mathrm{MNC}\left(\sim 6^{\circ}\right)$. 


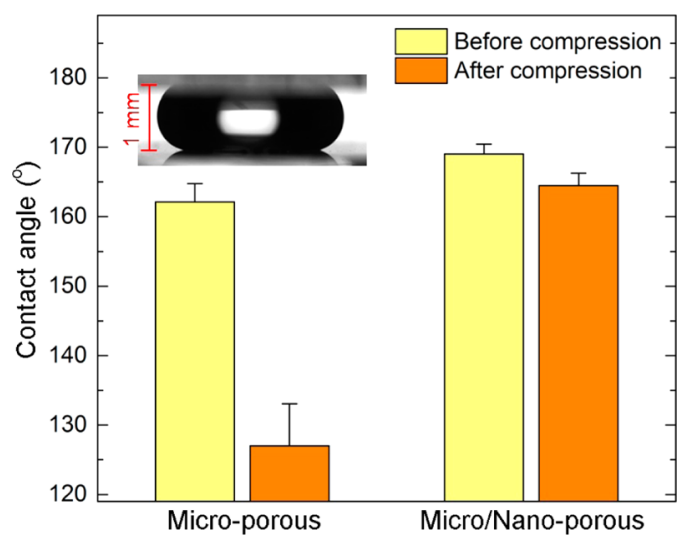

Figure 4. Droplet squeezing between two identical surfaces. Contact angles of surfaces before and after compression. The inset shows the squeezed water droplet between two MNC surfaces. There is only a slight decrease in the contact angle of the MNC after compression. The large decrease in the MC shows loss of the superhydrophobic property.

The excellent Cassie state stability of the MNC can be attributed to its double-layer structure (Scheme 1). For the $\mathrm{MC}$, the contact line gradually slides down from the walls of microporous structures with increasing pressure; after a critical pressure difference, the contact line touches the hydrophilic glass substrate and pins to the surface instantly (transition to the Wenzel state). On the other hand, for the MNC the bottom NC layer prevents interaction between the glass substrate and contact line. Under a critical pressure difference, the contact line must touch the NC surface, and at that point, transition to the nano-Cassie state from the initial micro-Cassie state can be expected. ${ }^{36}$ After removing the pressure, contact line can partially recover to the initial micro-Cassie state. The increase in contact-angle hysteresis and the sliding angle and the slight decrease in the apparent contact angle support the proposed partial transition model between nano- and micro-Cassie states.

Evaporation Experiments. During evaporation of water droplets from superhydrophobic surfaces, Laplace pressures as high as a few thousand pascal can be generated because of size
Scheme 1. Schematic Representation of the Proposed Model for the Excellent Cassie State Stability of the MNC Coating ${ }^{a}$

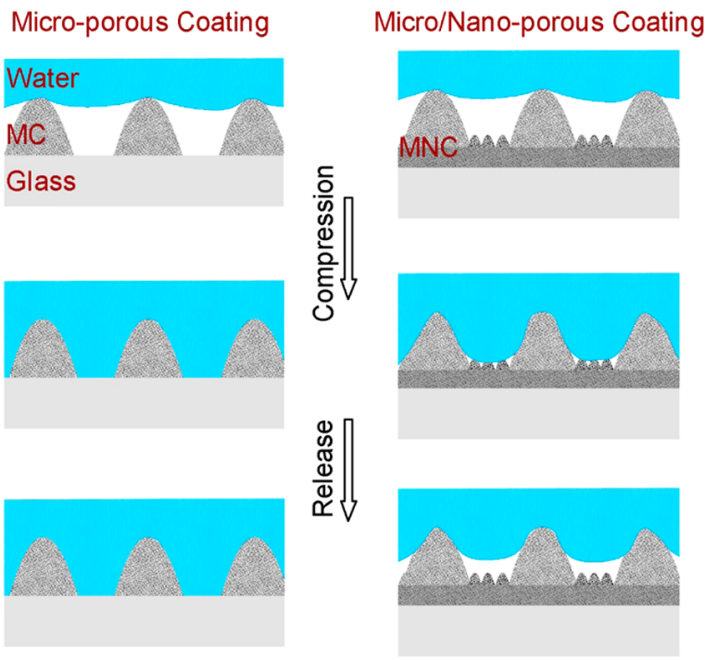

${ }^{a}$ For the MC, the contact line gradually slides down from the walls of microporous structures with increasing pressure; after a critical pressure difference, the contact line touches the hydrophilic glass substrate and pins to the surface instantly (transition to the Wenzel state). On the other hand, for the MNC, the bottom NC layer prevents interaction between the glass substrate and contact line.

reduction of the droplet, which is around 1 order of magnitude larger than the pressure difference generated during compression experiments. ${ }^{49,50}$ Therefore, to further demonstrate the Cassie state stability of the MNC, we performed evaporation experiments by placing water droplets $(6 \mu \mathrm{L})$ to the surfaces and allowing their evaporation at ambient conditions (approximately at $22{ }^{\circ} \mathrm{C}$ and $23 \%$ humidity).

Figure 6a shows time-dependent evaluation of the shape of water droplets on the MC and MNC. A water droplet on the MC lost its spherical shape gradually during evaporation and pinned to the surface, which indicates the Wenzel transition. On the other hand, a water droplet on the MNC preserved its spherical shape even at the last stages of evaporation, which
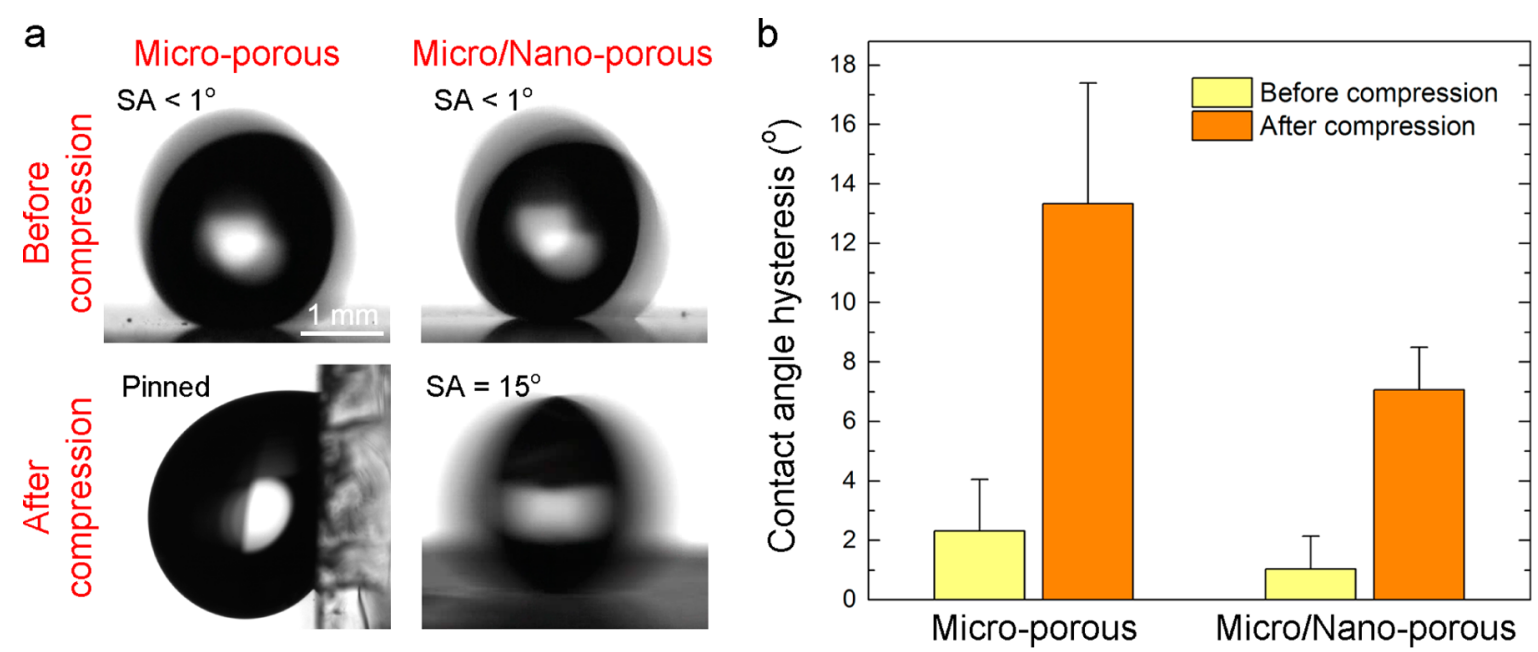

Figure 5. Droplet squeezing between two identical surfaces. (a) Sliding angles of surfaces before and after compression experiments for the MC (left column) and MNC (right column). A droplet on the MC sticks to the surface after compression due to Wenzel transition, whereas a water droplet on the MNC easily rolled off from the surface at $15^{\circ}$ tilting angle, indicating that the water droplet is still at the Cassie state. (b) Contact-angle hysteresis of the surfaces before and after compression. The increase in contact-angle hysteresis is significantly larger for the MC. 

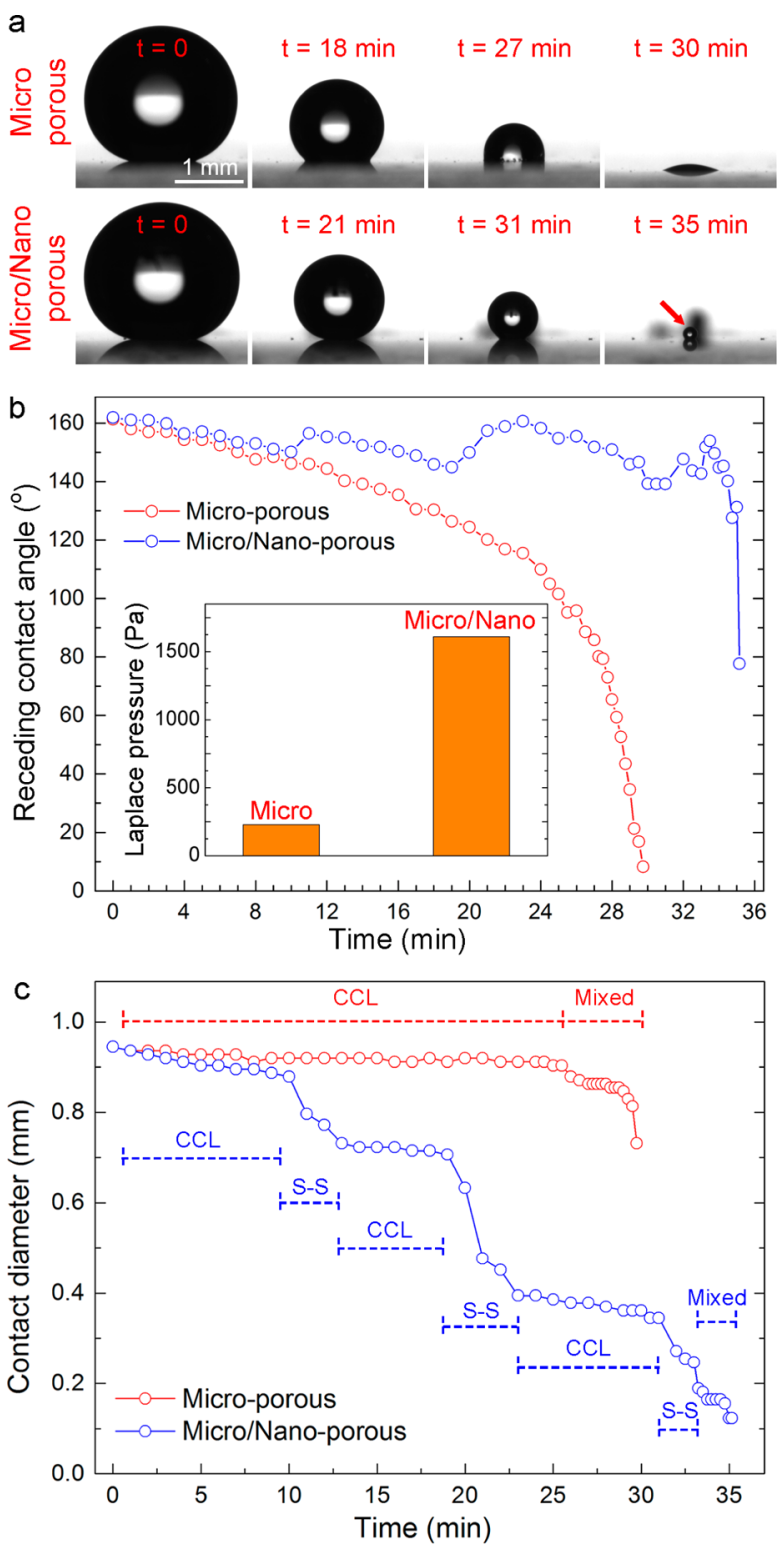

Figure 6. Evaporation experiments. (a) Photographs of water droplets sitting on the MC (upper row) and MNC (lower row) at different instants of the evaporation process. Wenzel transition was observed for a droplet sitting on the MC at around $18 \mathrm{~min}$. A water droplet on the MNC, on the other hand, preserved its spherical shape even at the final stages of evaporation, indicating the excellent Cassie stability of this coating. (b) Contact angles of the surfaces during evaporation experiment. The inset shows the generated Laplace pressures at the point where contact angles of the surfaces decrease below $120^{\circ}$. (c) Change of the water droplet contact-line diameter during evaporation. A CCL mode was observed for the MC. For the MNC, sudden decreases in the contact-line diameter were observed. This behavior, where the contact line of the droplet retracts and the contact angle increases suddenly, is known as stick-slip $(\mathrm{S}-\mathrm{S})$ behavior, referring to successive "pinning-depinning" of the droplet.

shows that the water droplet was still at the Cassie state. Figure $6 \mathrm{~b}$ shows the apparent contact-angle change of the surfaces during evaporation. The contact angle of the water droplet on the MC continuously decreased to $120^{\circ}$ in about $22 \mathrm{~min}$ and sharply decreased to almost $0^{\circ}$ after approximately $30 \mathrm{~min}$, where the droplet completely evaporated. The contact angle of the water droplet on the MNC decreased similarly to the MC for the first $10 \mathrm{~min}$. However, at this point, a jump in the contact angle occurred and it almost completely recovered to its initial value. Then it started to decrease again, and a second jump occurred after 19 min. Similarly, a third jump was observed after $31 \mathrm{~min}$. At the 35 th minute, the size of the droplet largely reduced, and after approximately $10 \mathrm{~s}$, it completely evaporated. The reason for this behavior will be discussed in more detail below. Note that the time required for complete evaporation of a droplet is slightly lower on the MC compared to the MNC, which is due to the increased evaporation rate after Wenzel transition (i.e., improved wettability) for this coating. To compare the Cassie state stability of coatings during droplet evaporation, we calculated the Laplace pressures ${ }^{27}(\Delta P=2 \gamma / R$, where $\gamma$ is the surface tension of water and $R$ is the droplet radius with a spherical shape) at the points where the contact angle of the water droplets decreases to around $120^{\circ}(t=21$ and $35 \mathrm{~min}$ for the $\mathrm{MC}$ and $\mathrm{MNC}$, respectively; Figure $6 \mathrm{~b}$, inset). The Laplace pressure at this point for the $\mathrm{MNC}(1620 \mathrm{~Pa})$ is almost 7-fold higher than that of the MC $(248 \mathrm{~Pa})$. We note that the Cassie state stability of the MNC is even better than that of the Lotus leaf, where the same transition was observed slightly above 400 $\mathrm{Pa}^{27}$ The difference between the pressure values where the contact angles of the MC and MNC surfaces and Lotus leaf reached $120^{\circ}$ demonstrates the enhanced Cassie state stability of the MNC surface. The contact-angle decrease rate of the MNC surface is much slower compared to that of both the MC surface and Lotus leaf, and Wenzel transition occurs at much later stages of evaporation.

For droplet evaporation experiments, four evaporation behaviors were defined in the previous studies: ${ }^{29,51-58}$ (i) the constant-contact-line (CCL) state, where the contact-line diameter is constant and the contact angle is decreasing, (ii) the constant-contact-angle (CCA) state, where the contact angle is constant and the contact-line diameter is decreasing, (iii) the stick-slip $(\mathrm{S}-\mathrm{S})$ behavior, where the droplet baseline retracts step by step, resulting in sudden increases in the contact angle, ${ }^{58}$ and (iv), the mixed state, where both the contact angle and contact-line diameter are decreasing. To identify the evaporation modes of our surfaces, we investigated the contact-line diameters of droplets during evaporation (Figure 6c). For the MC, the CCL mode was observed in the first $25 \mathrm{~min}$, where the diameter of the contact line is almost constant and the apparent contact angle is constantly decreasing (Figure $6 \mathrm{~b}, \mathrm{c}$ ). Also, at the last stages of evaporation, a mixed mode was observed. For the MNC, more complicated evaporation modes were observed (Figure 6c). In the first 10 min, a CCL mode similar to that for the MC was observed. However, after this point, a sudden decrease in the diameter of the contact line was observed. Note that a sudden decrease in the contact-line diameter occurred at the same time with a jump in the contact angle. This behavior has been observed previously on rough hydrophobic surfaces and is called as "stick-slip", referring to successive "pinning-depinning" of droplets during evaporation. ${ }^{33,59-61}$ As in contact-angle measurements, there are three abrupt changes in the baseline diameter of the MNC where the S-S behavior was observed, and between these jumps, the contact line can be approximated to be constant (CCL mode). Also, at the last stages of evaporation, the mixed mode was observed.

The CCL modes observed for both coatings can be explained by analyzing the forces at the triple contact line. ${ }^{29,62}$ At $t=0$, a droplet is in the equilibrium and the contact line is fixed. Then 
water starts to evaporate and therefore the droplet volume is continuously decreasing, which results in a decrease in the contact angle. The decrease in the contact angle disturbs the equilibrium between forces acting on the droplet and generates a radial force toward the center of the drop. This force (i.e., depinning force) retracts the contact line and recovers the initial contact angle. The depinning force $\left(F_{D}\right)$ can be expressed as $^{62}$

$$
F_{\mathrm{D}}=2 R_{\mathrm{b}} \sigma_{\lg }\left(\cos \theta_{t}-\cos \theta_{\mathrm{i}}\right)
$$

where $R_{\mathrm{b}}$ is contact-line radius, $\sigma_{\mathrm{lg}}$ is the surface tension of water, $\theta_{t}$ is the contact angle at a certain interval during evaporation, and $\theta_{\mathrm{i}}$ is the initial contact angle. Figure 7a shows
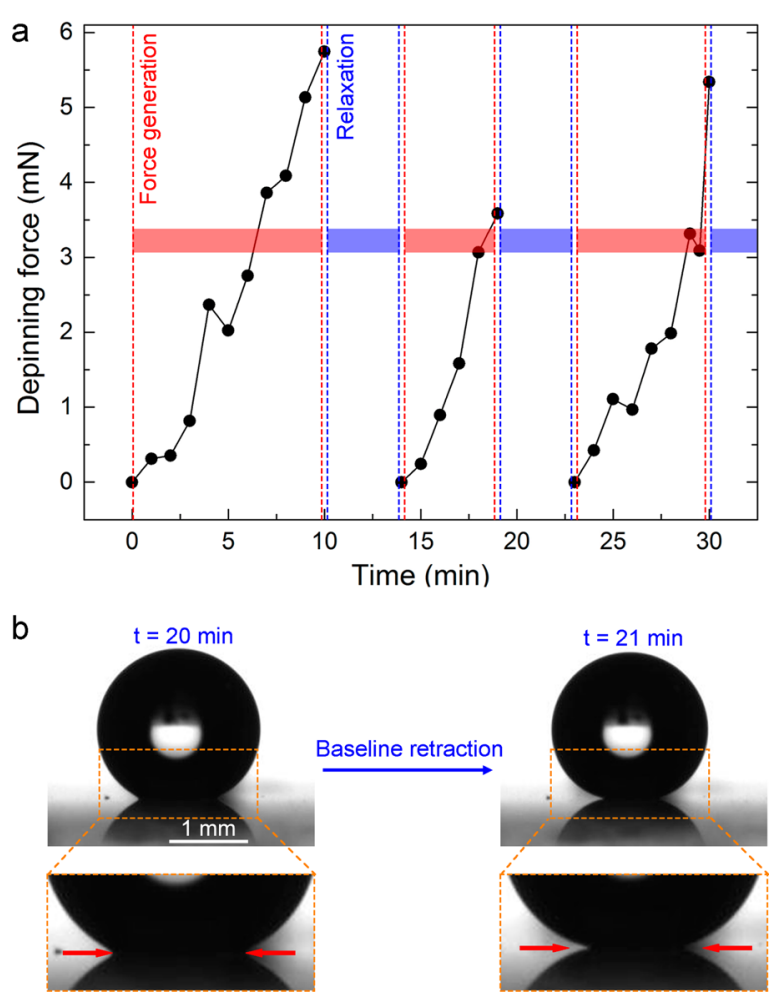

Figure 7. Force generation and relaxation of the contact line for the MNC. (a) Depinning force generation and its relaxation during evaporation. Red areas indicate the force generation, and blue areas indicate the relaxation regions. (b) Baseline retraction for the MNC between 20 and $21 \mathrm{~min}$. After baseline retraction, the baseline diameter significantly decreased and the contact angle increased.

the depinning force generated during the evaporation process for the MNC. We observed that, during evaporation, first a depinning force around $5 \mathrm{mN}$ was generated and then the droplet relaxed by retraction of the contact line, and this cycle was repeated three times. Figure $7 \mathrm{~b}$ shows the contact-line retraction between the 20th and 21th minutes. After contactline retraction, the apparent contact angle of the droplet reaches its initial value, which indicates total relaxation. Note that no relaxation was observed for the MC even at depinning forces larger than $100 \mathrm{mN}$, which shows that the water droplet on the MC strongly pins to the surface during evaporation (see the SI, Figure S2).

\section{CONCLUSION}

The present work demonstrates a facile method to fabricate transparent and superhydrophobic ormosil surfaces with robust
Cassie states of wetting. We prepared two superhydrophobic surfaces on glass substrates: (i) a single-layer microporous coating and (ii) a double-layer coating with nanoporous bottom and microporous top layers. The stability of the Cassie state of the coatings against the external pressure was investigated with droplet compression and evaporation experiments. It was observed that a droplet sitting on a single-layer coating can easily transform to a sticky Wenzel state from a roll-off Cassie state under external pressures as low as approximately $80 \mathrm{~Pa}$. On the other hand, Cassie to Wenzel state transition was observed at around $1600 \mathrm{~Pa}$ for a double-layer micro/ nanoporous coating, which is almost 4-fold higher than the transition pressure for a Lotus leaf ${ }^{27}$ (slightly above $400 \mathrm{~Pa}$ ). The observed extreme stability of the Cassie state in a micro/ nanoporous coating can be attributed to its double-layer porous structure. With increasing external pressure, the contact line of a droplet in the Cassie state gradually slides down from the walls of a microporous top layer, and after a critical pressure, the contact line touches the nanoporous bottom layer. At that point, transition to the nano-Cassie state from the micro-Cassie state can be expected. After removal of the pressure, the contact line can partially recover to the initial micro-Cassie state. However, for a single-layer coating, under external pressure, the contact line touches the hydrophilic glass substrate (instead of the hydrophobic nanoporous layer) and strongly pins to the surface (transition to the Wenzel state). The outcomes of this study may provide researchers an adequate ground for designing robust superhydrophobic surfaces for outdoor and underwater applications where surfaces are exposed to high external pressures.

\section{ASSOCIATED CONTENT}

Supporting Information

Additional SEM images of the surfaces and a graph showing the depinning force generation during evaporation for the MC. This material is available free of charge via the Internet at http://pubs.acs.org.

\section{AUTHOR INFORMATION}

\section{Corresponding Author}

*Phone: +90 312290 2513. E-mail: bayindir@nano.org.tr.

Notes

The authors declare no competing financial interest.

\section{ACKNOWLEDGMENTS}

U.T. and A.Y. are supportd by TÜBİTAK-BIDEB graduate student fellowship. This work is supported by TÜBİTAK under Project 111T696. M.B. acknowledges partial support from the Turkish Academy of Sciences.

\section{REFERENCES}

(1) Quere, D. Surface Chemistry: Fakir Droplets. Nat. Mater. 2002, $1,14-15$.

(2) Lafuma, A.; Quere, D. Superhydrophobic States. Nat. Mater. 2003, 2, 457-460.

(3) Wang, S.; Jiang, L. Definition of Superhydrophobic States. Adv. Mater. 2007, 19, 3423-3424.

(4) Yildirim, A.; Khudiyev, T.; Daglar, B.; Budunoglu, H.; Okyay, A. K.; Bayindir, M. Superhydrophobic and Omnidirectional Antireflective Surfaces from Nanostructured Ormosil Colloids. ACS Appl. Mater. Interfaces 2013, 5, 853-860.

(5) Deng, X.; Mammen, L.; Zhao, Y.; Lellig, P.; Muellen, K.; Li, C.; Butt, H.-J.; Vollmer, D. Transparent, Thermally Stable and 
Mechanically Robust Superhydrophobic Surfaces Made from Porous Silica Capsules. Adv. Mater. 2011, 23, 2962-2965.

(6) Park, Y. B.; Im, H.; Im, M.; Choi, Y. K. Self-Cleaning Effect of Highly Water-Repellent Microshell Structures for Solar Cell Applications. J. Mater. Chem. 2011, 21, 633-636.

(7) McHale, G.; Newton, M. I.; Shirtcliffe, N. J. Immersed Superhydrophobic Surfaces: Gas Exchange, Slip and Drag Reduction Properties. Soft Matter 2010, 6, 714-719.

(8) Poetes, R.; Holtzmann, K.; Franze, K.; Steiner, U. Metastable Underwater Superhydrophobicity. Phys. Rev. Lett. 2010, 105, 166104.

(9) Barkhudarov, P. M.; Shah, P. B.; Watkins, E. B.; Doshi, D. A.; Brinker, C. J.; Majewski, J. Corrosion Inhibition Using Superhydrophobic Films. Corros. Sci. 2008, 50, 897-902.

(10) Oner, D.; McCarthy, T. J. Ultrahydrophobic Surfaces. Effects of Topography Length Scales on Wettability. Langmuir 2000, 16, 77777782 .

(11) Shirtcliffe, N. J.; McHale, G.; Newton, M. I.; Chabrol, G.; Perry, C. C. Dual-Scale Roughness Produces Unusually Water-Repellent Surfaces. Adv. Mater. 2004, 16, 1929-1932.

(12) Zhang, X. S.; Zhu, F. Y.; Han, M. D.; Sun, X. M.; Peng, X. H.; Zhang, H. X. Self-Cleaning Poly(dimethylsiloxane) Film with Functional Micro/Nano Hierarchical Structures. Langmuir 2013, 29, 10769-10775.

(13) Budunoglu, H.; Yildirim, A.; Guler, M. O.; Bayindir, M. Highly Transparent, Flexible, and Thermal Stable Superhydrophobic ORMOSIL Aerogel Thin Films. ACS Appl. Mater. Interfaces 2011, 3, $539-545$.

(14) Hikita, M.; Tanaka, K.; Nakamura, T.; Kajiyama, T.; Takahara, A. Super-Liquid-Repellent Surfaces Prepared by Colloidal Silica Nanoparticles Covered with Fluoroalkyl Groups. Langmuir 2005, 21, 7299-7302.

(15) Xu, Q. F.; Wang, J. N.; Sanderson, K. D. Organic-Inorganic Composite Nanocoatings with Superhydrophobicity, Good Transparency, and Thermal Stability. ACS Nano 2010, 4, 2201-2209.

(16) Erbil, H. Y.; Demirel, A. L.; Avci, Y.; Mert, O. Transformation of a Simple Plastic Into a Superhydrophobic Surface. Science 2003, 299, 1377-1380.

(17) Acatay, K.; Simsek, E.; Ow-Yang, C.; Menceloglu, Y. Z. Tunable, Superhydrophobically Stable Polymeric Surfaces by Electrospinning. Angew. Chem., Int. Ed. 2004, 43, 5210-5213.

(18) Ma, M.; Hill, R. M.; Lowery, J. L.; Fridrikh, S. V.; Rutledge, G. C. Electrospun Poly(styrene-block-dimethylsiloxane) Block Copolymer Fibers Exhibiting Superhydrophobicity. Langmuir 2005, 21, 55495554.

(19) Zhai, L.; Cebeci, F. C.; Cohen, R. E.; Rubner, M. F. Stable Superhydrophobic Coatings from Polyelectrolyte Multilayers. Nano Lett. 2004, 4, 1349-1353.

(20) Bravo, J.; Zhai, L.; Wu, Z. Z.; Cohen, R. E.; Rubner, M. F. Transparent Superhydrophobic Films Based on Silica Nanoparticles. Langmuir 2007, 23, 7293-7298.

(21) Gao, L.; Mccarthy, T. J. A Perfectly Hydrophobic Surface $\left(\theta_{\mathrm{A}} /\right.$ $\left.\theta_{\mathrm{R}}=180^{\circ} / 180^{\circ}\right)$. J. Am. Chem. Soc. 2006, 128, 9052-9053.

(22) Liu, X.; He, J. One-Step Hydrothermal Creation of Hierarchical Microstructures Toward Superhydrophilic and Superhydrophobic Surfaces. Langmuir 2009, 25, 11822-11826.

(23) Balu, B.; Breedveld, V.; Hess, D. W. Fabrication of "Roll-off" and "Sticky" Superhydrophobic Cellulose Surfaces via Plasma Processing. Langmuir 2008, 24, 4785-4790.

(24) Whyman, G.; Bormashenko, E. How to Make the Cassie Wetting State Stable? Langmuir 2011, 27, 8171-8176.

(25) Bobji, M. S.; Kumar, S. V.; Asthana, A.; Govardhan, R. N. Underwater Sustainability of the "Cassie" State of Wetting. Langmuir 2009, 25, 12120-12126.

(26) Papadopoulos, P.; Mammen, L.; Deng, X.; Vollmer, D.; Butt, H.-J. How Superhydrophobicity Breaks Down. Proc. Natl. Acad. Sci. U.S.A. 2013, 110, 3254-3258.

(27) Cha, T. G.; Yi, J. W.; Moon, M. W.; Lee, K. R.; Kim, H. Y. Nanoscale Patterning of Microtextured Surfaces to Control Superhydrophobic Robustness. Langmuir 2010, 26, 8319-8326.
(28) Susarrey-Arce, A.; Marin, A. G.; Nair, H.; Lefferts, L.; Gardeniers, J. G. E.; Lohse, D.; Van-Houselt, A. Absence of an Evaporation-Driven Wetting Transition on Omniphobic Surfaces. Soft Matter 2012, 8, 9765-9770.

(29) Chen, X.; Ma, R.; Li, J.; Hao, C.; Guo, W.; Luk, B. L.; Li, S. C.; Yao, S.; Wang, Z. Evaporation of Droplets on Superhydrophobic Surfaces: Surface Roughness and Small Droplet Size Effects. Phys. Rev. Lett. 2012, 109, 116101.

(30) Forsberg, P.; Nikolajeff, F.; Karlsson, M. Cassie-Wenzel and Wenzel-Cassie Transitions on Immersed Superhydrophobic Surfaces Under Hydrostatic Pressure. Soft Matter 2011, 7, 104-109.

(31) Gnanappa, A. K.; Papageorgiou, D. P.; Gogolides, E.; Tserepi, A.; Papathanasiou, A. G.; Boudouvis, A. G. Hierarchical, Plasma Nanotextured, Robust Superamphiphobic Polymeric Surfaces Structurally Stabilized Through a Wetting-Drying Cycle. Plasma Processes Polym. 2012, 9, 304-315.

(32) Kwon, Y.; Patanker, N.; Choi, J.; Lee, J. Design of Surface Hierarchy for Extreme Hydrophobicity. Langmuir 2009, 25, 61296136.

(33) McHale, G.; Aqil, S.; Shirtcliffe, N. J.; Newton, M. I.; Erbil, H. Y. Analysis of Droplet Evaporation on a Superhydrophobic Surface. Langmuir 2005, 21, 11053-11060.

(34) Milionis, A.; Fragouli, D.; Martiradonna, L.; Anyfantis, G. C.; Cozzoli, P. D.; Bayer, I. S.; Athanassiou, A. Spatially Controlled Surface Energy Traps on Superhydrophobic Surfaces. ACS Appl. Mater. Interfaces 2013, 6, 1036-1043.

(35) Tsai, P.; Lammertink, R. G. H.; Wessling, M.; Lohse, D. Evaporation-Triggered Wetting Transition for Water Droplets upon Hydrophobic Microstructures. Phys. Rev. Lett. 2010, 104, 116102.

(36) Verho, T.; Korhonen, J. T.; Sainiemi, L.; Jokinen, V.; Bower, C.; Franze, K.; Franssila, S.; Andrew, P.; Ikkala, O.; Ras, R. H. A. Reversible Switching Between Superhydrophobic States on a Hierarchically Structured Surface. Proc. Natl. Acad. Sci. U.S.A. 2012, 109, 10210-10213.

(37) Nosonovsky, M. Multiscale Roughness and Stability of Superhydrophobic Biomimetic Interfaces. Langmuir 2007, 23, 31573161.

(38) Yao, X.; Chen, Q. W.; Xu, L.; Li, Q. K.; Song, Y. L.; Gao, X. F.; Quere, D.; Jiang, L. Bioinspired Ribbed Nanoneedles with Robust Superhydrophobicity. Adv. Funct. Mater. 2010, 20, 656-662.

(39) Tuteja, A.; Choi, W.; Ma, M.; Mabry, J. M.; Mazzella, S. A.; Rutledge, G. C.; McKinley, G. H.; Cohen, R. E. Designing Superoleophobic Surfaces. Science 2007, 318, 1618-1622.

(40) Tuteja, A.; Choi, W.; McKinley, G. H.; Cohen, R. E.; Rubner, M. F. Design Parameters for Superhydrophobicity and Superoleophobicity. MRS Bull. 2008, 33, 752-758.

(41) Yildirim, A.; Budunoglu, H.; Yaman, M.; Guler, M. O.; Bayindir, M. Template Free Preparation of Nanoporous Organically Modified Silica Thin Films on Flexible Substrates. J. Mater. Chem. 2011, 21, 14830-14837.

(42) Bravo, J.; Zhai, L.; Wu, Z. Z.; Cohen, R. E.; Rubner, M. F. Transparent Superhydrophobic Films Based on Silica Nanoparticles. Langmuir 2007, 23, 7293-7298.

(43) Nakajima, A.; Hashimoto, K.; Watanabe, T.; Takai, K.; Yamauchi, G.; Fujishima, A. Transparent Superhydrophobic Thin Films with Self-Cleaning Properties. Langmuir 2000, 16, 7044-7047.

(44) Manca, M.; Cannavale, A.; De Marco, L.; Aricò, A. S.; Cingolani, R.; Gigli, G. Durable Superhydrophobic and Antireflective Surfaces by Trimethylsilanized Silica Nanoparticles-Based Sol-Gel Processing. Langmuir 2009, 25, 6357-6362.

(45) Ling, X. Y.; Phang, I. Y.; Vancso, G. J.; Huskens, J.; Reinhoudt, D. N. Stable and Transparent Superhydrophobic Nanoparticle Films. Langmuir 2009, 25, 3260-3263.

(46) Cho, K. L.; Liaw, I. I.; Wu, A. H. F.; Lamb, R. N. Influence of Roughness on a Transparent Superhydrophobic Coating. J. Phys. Chem. C 2010, 114, 11228-11233.

(47) Raut, H. K.; Ganesh, V. A.; Nair, A. S.; Ramakrishna, S. AntiReflective Coatings: A Critical, In-Depth Review. Energy Environ. Sci. 2011, 4, 3779-3804. 
(48) Callies, M.; Quéré, D. On Water Repellency. Soft Matter 2005, $1,55-61$.

(49) Li, L. H.; Chen, Y. Superhydrophobic Properties of Nonaligned Boron Nitride Nanotube Films. Langmuir 2010, 26, 5135-5140.

(50) Choi, C. H.; Kim, C.-J. Droplet Evaporation of Pure Water and Protein Solution on Nanostructured Superhydrophobic Surfaces of Varying Heights. Langmuir 2009, 25, 7561-7567.

(51) Xu, W.; Leeladhar, R.; Kang, Y. T.; Choi, C. H. Evaporation Kinetics of Sessile Water Droplets on Micropillared Superhydrophobic Surfaces. Langmuir 2013, 29, 6032-6041.

(52) Dash, S.; Garimella, S. V. Droplet Evaporation Dynamics on a Superhydrophobic Surface with Negligible Hysteresis. Langmuir 2013, 29, 10785-10795.

(53) Picknett, R. G.; Bexon, R. The Evaporation of Sessile or Pendant Drops in Still Air. J. Colloid Interface Sci. 1977, 61, 336-350.

(54) Birdi, K. S.; Vu, D. T.; Winter, A. A Study of the Evaporation Rates of Small Water Drops Placed on a Solid Surface. J. Phys. Chem. 1989, 93, 3702-3703.

(55) Bourges-Monnier, C.; Shanahan, M. E. R. Influence of Evaporation on Contact Angle. Langmuir 1995, 11, 2820-2829.

(56) Erbil, H. Y.; McHale, G.; Newton, M. I. Drop Evaporation on Solid Surfaces: Constant Contact Angle Mode. Langmuir 2002, 18, 2636-2641.

(57) Erbil, H. Y. Evaporation of Pure Liquid Sessile Drops: A Review. Adv. Colloid Interface Sci. 2012, 170, 67-86.

(58) Shanahan, M. E. R. Simple Theory of "Stick-Slip" Wetting Hysteresis. Langmuir 1995, 11, 1041-1043.

(59) Extrand, C. W.; Kumagai, Y. An Experimental Study of Contact Angle Hysteresis. J. Colloid Interface Sci. 1997, 191, 378-383.

(60) McHale, G.; Rowan, S. M.; Newton, M. I.; Banerjee, M. K. Evaporation and the Wetting of a Low-Energy Solid Surface. J. Phys. Chem. B 1998, 102, 1964-1967.

(61) Orejon, D.; Sefiane, K.; Shanahan, M. E. R. Stick-Slip of Evaporating Droplets: Substrate Hydrophobicity and Nanoparticle Concentration. Langmuir 2011, 27, 12834-12843.

(62) Angelis, F. D.; Gentile, F.; Mecarini, F.; Das, G.; Moretti, M.; Candeloro, P.; Coluccio, M. L.; Cojoc, G.; Accardo, A.; Liberale, C.; Zaccaria, R. P.; Perozziello, G.; Tirinato, L.; Toma, A.; Cuda, G.; Cingolani, R.; Di Fabrizio, E. Breaking the Diffusion Limit with SuperHydrophobic Delivery of Molecules to Plasmonic Nanofocusing SERS Structures. Nat. Photonics 2011, 5, 682-687. 